\title{
La Oficina del Historiador de La Habana al rescate del patrimonio bibliográfico
}

\author{
Grisel Terrón Quintero
}

\section{Introducción}

Históricamente el espacio conocido como La Habana Vieja fue el centro político del país y sede de los gobiernos colonial e interventor, provincial y municipal y de buena parte de instituciones de alcance nacional como varios ministerios. En este entorno se sitúa la Oficina del Historiador de La Habana, entidad en principio provincial pero cuya repercusión sin dudas trasciende sus fronteras geográficas y jurídicas.

La Oficina del Historiador de la ciudad de La Habana ha constituido, desde su fundación en 1938, una institución de vanguardia. Desde que en 1935 Emilio Roig de Leuchsenring fuera nombrado Historiador de la Ciudad, incluso sin existir la Oficina del Historiador como institución, los libros y documentos ocuparon gran parte de la gestión de la entidad. Junto al surgimiento de la Oficina, nacieron el Archivo Histórico y la Biblioteca Histórica Cubana y Americana, ambos en un valiente intento de salvar "papeles" del paso del tiempo y de los hombres.

La Biblioteca Histórica surgió, además, como acto contestatario y entregado de democratización de la cultura pues al alcance de todos se pusieron bibliotecas particulares enteras. De la misma manera, la Oficina nació, no solo con la vocación de rescate de la bibliografía cubana publicada, sino con la disposición de hacerla proliferar y por eso creó un sistema de publicaciones que logró salvar a Cuba de la pérdida de una parte de su memoria de las guerras de Independencia y de la República.

\section{Desarrollo}

Los primeros años de la década de los años sesenta, impactados por el Triunfo de la Revolución y el ímpetu rebelde, si bien mostraron la preocupación del nuevo gobierno por la cultura', estaban cargados de desorientación respecto a los valores culturales de inmuebles, propiedades, bibliotecas, papeles y otros exponentes de lo que podía ser el "patrimonio”. Bajo este espíritu desaparecieron libros y documentos valiosos.

La Oficina del Historiador sostenida por Emilio Roig no escapó de esto: muchos libros y documentos Imprenta Nacional que publica El Quijote y se celebran eventos como el Festival del Libro y la Lectura, el Primer Festival del Libro Revolucionario, el Congreso de Escritores y Artistas (1961) y la Primera Gran Feria Nacional del Libro (1966).

A mí me tocó ser testigo del silencio que reinó cuando Emilito ya no estaba y se pensaba que la Oficina del Historiador de la Ciudad iría languideciendo mientras los bienes patrimoniales, muchos de ellos pertenecientes a su colección personal, salían hacia el Instituto de Historia, la 


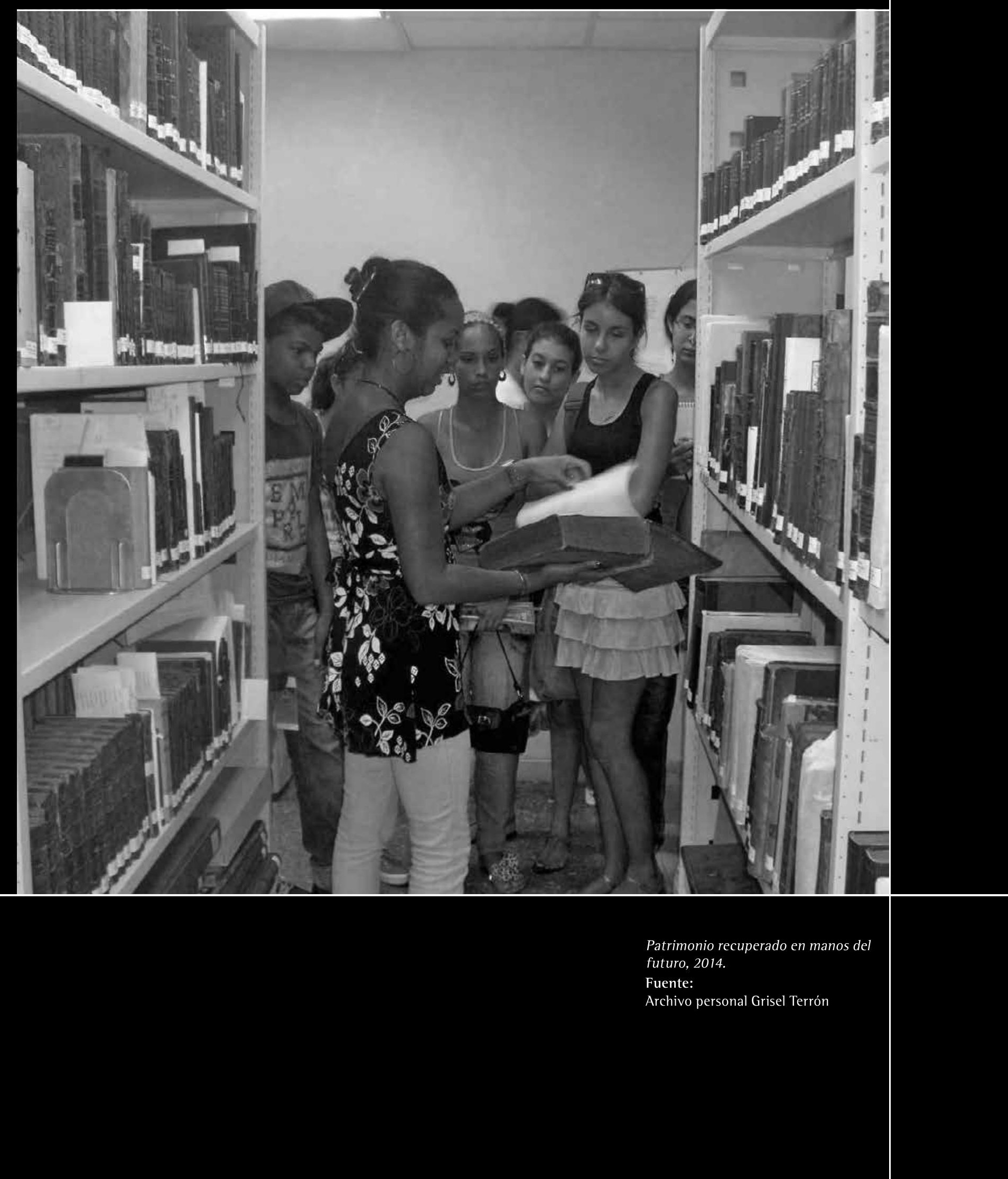




\section{La Oficina del Historiador de La Habana al rescate del patrimonio bibliográfico}

The Office of the Havana City Historian recovering the bibliographic heritage

A Oficina do Historiador de Habana recuperando o património bibliográfico

Grisel Terrón Quintero

grisel@patrimonio.ohc.cu

Doctora en Gestión del Patrimonio Cultural, Universidad de Granada. Ha cursado diversos postgrados y diplomados sobre patrimonio cultural, libros antiguos, tecnologías de la información y conservación de documentos en Cuba y Europa. Ha impartido conferencias dentro y fuera de Cuba y ha sido ponente de eventos nacionales y extranjeros. Además de dirigir la gestión del patrimonio documental en la Oficina del Historiador, ha realizado varias investigaciones sobre la gestión cultural enfocadas en el patrimonio documental, las colecciones que se atesoran en las bibliotecas y los archivos y sobre la interpretación del patrimonio. Cuenta con varias publicaciones.

Resumen

La Oficina del Historiador de la ciudad de La Habana constituye, desde su fundación en 1938, una institución de vanguardia capaz de mostrar una obra cultural y social que trasciende su espacio geográfico y jurídico. Desde sus inicios la Oficina del Historiador ensayó variantes novedosas que facilitaron el rescate del patrimonio. El "período especial", iniciado en los años noventa, reconfiguró las potestades del Historiador y su Oficina para garantizar un espacio de innovación que pusiera a salvo la cultura y el patrimonio. El trabajo analiza las alternativas empleadas para rescatar, preservar y difundir el patrimonio bibliográfico a partir de los años noventa.

Palabras clave: La Habana; bibliotecas; patrimonio bibliográfico; cultura

Abstract

The Office of the Havana City Historian has been, since its foundation in 1938, an institution of vanguard capable to show a cultural and social work that transcends its geographical and juridical space. From its beginnings the Historian Office tried alternative ways to achieve the preservation of the historical heritage. The special period initiated in the 1990 's reconfigured the authorities of the Historian Office to assure the innovation space aimed to save the cultural traditions and the patrimony. This work analyzes the alternatives that have been used since the 1990's to save, preserve and publicize the Bibliographic heritage.

Keywords: La Habana; libraries; bibliographical heritage; culture

Artículo de investigación

Recepción: 9 de marzo de 2015

Aceptación: 25 de noviembre de 2015

Disponible en línea: 31 de enero de 2016
Resumo

A oficina do Historiador da cidade de Habana constitui, desde a sua fundação em 1938, uma instituição de vanguarda capaz de mostrar uma obra cultural e social que transpõe o seu espaço geográfico e jurídico. Desde o inicio a Oficina do Historiador ensaiou várias novidades que facilitaram o resgate do património. 0 "período especial", iniciado nos anos noventa reconfigurou as potencialidades do historiador e a sua Oficina para garantir um espaço de inovação que pôs a salvo a cultura e o património. 0 trabalho analisa as alternativas empregues para resgatar, preservar e difundir o património bibliográfico a partir dos anos noventa.

Palavras-chave: Habana; biblioteca; património bibliográfico; cultural

doi:10.11144/Javeriana.apc28-2.ohhr 


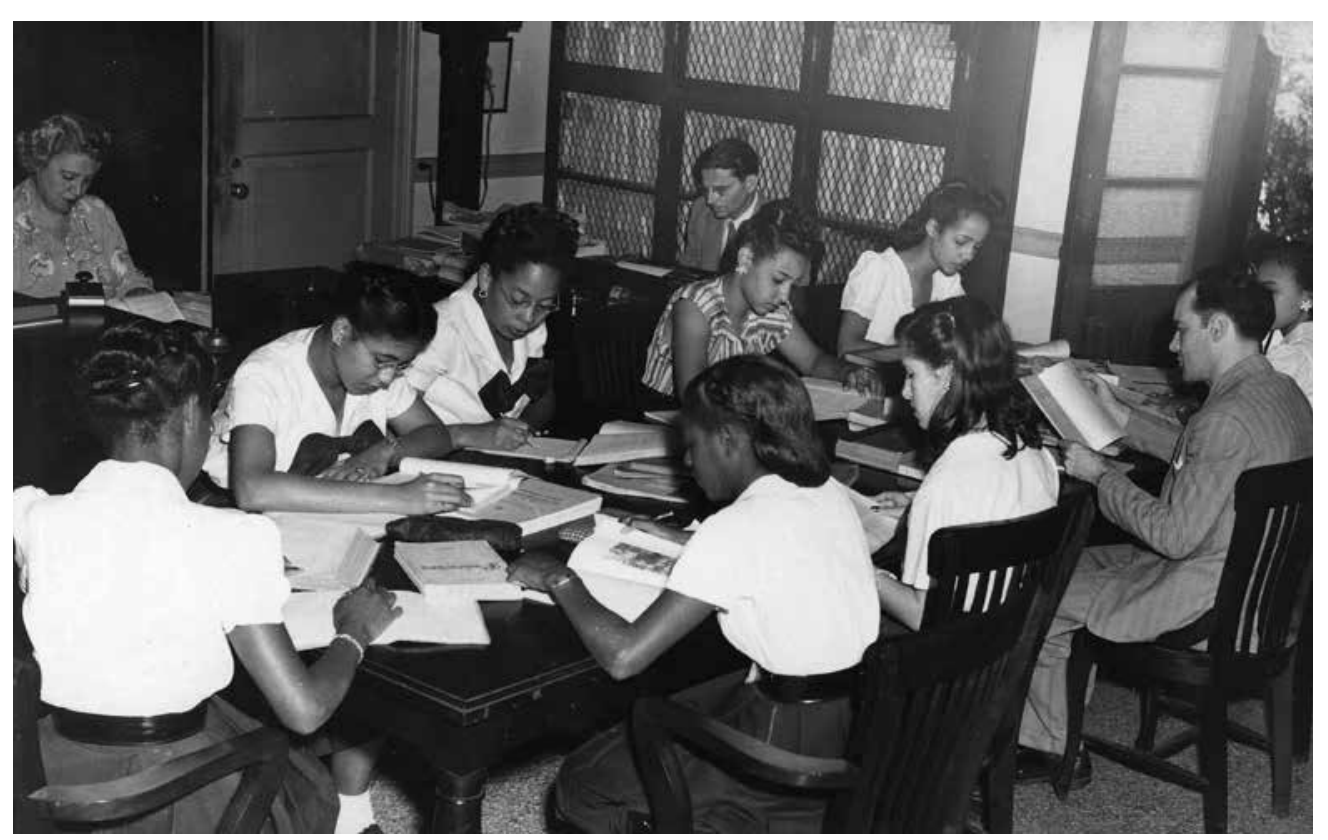

Biblioteca Nacional, la Academia de Ciencias, el Archivo Nacional [...]

A este último, por ejemplo, fueron a parar los grabados que le había regalado Conrado Massaguer, la colección de retratos de cubanos y extranjeros ilustres que habían visitado a Cuba [...] También salieron las cartas de Martí que el hijo de Manuel Mercado había donado y que Emilito conservaba al lado del buró, junto a sus libros personales, los cuales fueron enviados a la Biblioteca Nacional (Leal, 2009, p. 27).

La Biblioteca Histórica, creada a partir de las donaciones de notables intelectuales en las décadas de los años treinta y cuarenta fue prácticamente desmembrada a la muerte de Roig, pero la certera labor de Eusebio Leal al frente del empeño por rescatar la obra de su antecesor, logró devolver a sus predios la biblioteca primigenia.

\section{La década de los ochenta}

La inclusión de La Habana Vieja en la lista del Patrimonio de la Mundial con el número 27, en 1982, validó la obra rehabilitadora y abrió nuevas posibilidades de gestión.

Numerosas edificaciones fueron reconstruidas, rehabilitadas o restauradas y en ellas se fundaron o se instalaron en mejores condiciones, museos, bibliotecas, instituciones especializadas siempre en la esfera de la cultura con la sola excepción de los templos Habana. Plan Maestro, 1996, p. 2).

A partir de entonces, el Estado proveyó de fondos a la Oficina del Historiador de la Ciudad de La Habana para invertir en el proceso de restauración del Centro Histórico. Como complemento surgió un pequeño Departamento de Arquitectura para desarrollar proyectos de restauración de los inmuebles con un marcado carácter social.

De esta época data la Feria de la Catedral, donde se empezó a comercializar artesanía cubana lo que dotó al Centro Histórico de una dinámica sui génerisy un sello propio: en ella tenía cabida la iniciativa individual y el gusto diferenciado. También se desarrollaron experiencias como la Casa de la Natilla, Casa del Café con Leche en Obispo donde, por un precio módico, se podían encontrar ofertas gastronómicas inexistentes en otros lugares, todo esto en un entorno histórico cuyas ganancias se convertían en patrimonio rescatado.

Hasta 1990, llegaron a rescatarse más de 60 monumentos arquitectónicos y se reanimó la imagen urbana del Centro Histórico de La Habana Vieja, en un monto alrededor de 30 millones de pesos (Volta, 2010, p. 6).

$Y$ mientras se construía de alguna manera una forma particular de generar recursos, la Oficina del Historiador no descuidaba el patrimonio bibliográfico y la responsabilidad de conservar y (Oficina del Historiador de la Ciudad de La

Figura 1:

Biblioteca Histórica Cubana y Americana Francisco González del Valle en 1938.

Fuente:

Biblioteca Histórica 
difundir textos. Surgieron varias bibliotecas especializadas en el seno de museos y casas-museos creadas por las buenas voluntades de la Oficina y de muchos países que, a través de sus embajadas en La Habana, las proveyeron de textos especializados ${ }^{2}$. lgualmente se acogieron bibliotecas surgidas en años anteriores como la Biblioteca Fermín Valdés Domínguez de la Casa Natal José Martí creada en 1978.

\section{Los años noventa: período especial}

La caída del bloque socialista europeo marcó el inicio de la década de los noventa con la llegada de lo que se definió como "período especial". Sin dudas, la cultura sentiría el impacto de la crisis y, por supuesto, la intención de rehabilitar el Centro Histórico.

En 1990, apenas iniciado el tercero de los planes quinquenales, comienza a sentirse en el país el impacto de la caída del bloque socialista con el consiguiente agravamiento de la situación económica, lo cual provoca una encrucijada. Por una parte, el Estado Cubano, que hasta el momento había subvencionado centralmente la recuperación del Centro Histórico, no se encuentra en condiciones de continuar financiando esta actividad ante el declive de otros sectores aún más sensibles; por la otra, el propio Estado es consciente de la responsabilidad que significa salvar un patrimonio que no solo pertenece a los cubanos, sino a la humanidad (Oficina del Historiador de la Ciudad de La Habana, 1999, p. III).

Y en la inminencia de perder lo construido -en todo el sentido de la expresión- se inició un reajuste estructural del país que condujo a la redistribución de los recursos y al inicio de varios programas de desarrollo. Se promulgaron normativas para la entrada de capitales, se crearon empresas mixtas (con participación cubana y extranjera) y otros tipos de asociaciones. Igualmente, se inició una modalidad de gestión privada bajo el nombre de "trabajo por cuenta propia".

La Oficina del Historiador se inscribió en se crearon en la década de los ochenta están la Don Fernando Ortiz (1986) de la Casa de África, la del Gabinete de Arqueología (1987) y la Alfonso Reyes (1988) de la Casa de México.
La crisis económica de los '90 impone un nuevo reto para la salvaguarda de La Habana Vieja. A los ya reconocidos valores socioculturales del Centro Histórico ha de sumarse una nueva visión económica. El patrimonio puede ser productivo, y una eficiente explotación del mismo, siempre bajo una óptica cultural y de desarrollo social, permite dar continuidad a la obra rehabilitadora en su dimensión más integral [...] (Oficina del Historiador de la Ciudad de La Habana, 1999, p. IV).

El Decreto Ley dotaba a la Oficina del Historiador de un respaldo legal para promover un desarrollo sostenible a partir de la creatividad interna. En ese momento la Oficina dejó de ser una institución supeditada al Gobierno Provincial de la Ciudad y pasó directamente al Consejo de Estado. El artículo 4 personalizaba la dirección de la Oficina en la figura del Historiador de la Ciudad y lo ubicaba bajo la subordinación directa del Presidente de los Consejos de Estado y de Ministros. Dos años después, en noviembre de 1995, con la proclamación del Acuerdo 2951, el Centro Histórico se declaró zona de alta significación para el turismo, y se ampliaron las facultades de la Oficina.

Con las dos normas -en especial la del año 1993- se establecieron jurídicamente en Cuba lo que de hecho serían las bases de un nuevo modelo en la gestión y el manejo de los centros históricos en el país. En primer término, se ampliaban las facultades del Historiador de la Ciudad, y en segundo, se le permitía autofinanciar su labor mediante la obtención y administración de sus recursos económicos.

El artículo 6 del Decreto definió claramente las funciones de la Oficina del Historiador y le dio cobertura para "Preservar la memoria material y espiritual de la capital de la República como expresión de la historia nacional, divulgándola y honrándola por todos los medios de difusión, naturales y técnico-científicos y por su acción continúa sobre estos bienes de la nación" (Cuba, Ministerio de Justicia, 1993).

Con este amparo se inició la generación de recursos financieros propios y la cooperación internacional, y se estableció un gravamen fijado por el Ministerio de Finanzas y Precios a las entidades no subordinadas a la Oficina del Historiador enclavadas en la zona priorizada para la conservación. 
Todas estas variantes tuvieron, y tienen, amplia repercusión en el rescate y gestión del patrimonio bibliográfico y en el mantenimiento y superación de la práctica tradicional en este ámbito en la Oficina del Historiador.

Una vez más la Oficina del Historiador incorporó sectores y gestiones excluidas muchas veces y brindó al mercado del libro antiguo un espacio regulado para la captación de ingresos para la recuperación del patrimonio. La Feria de Libros Viejos de la Plaza de Armas y la Librería El Navío brindaron la plataforma para el rescate de libros, revistas y documentos que se hubieran perdido para siempre en la precariedad de la vida cubana de los años noventa. Ambas fórmulas establecieron un flujo que generaba recursos para la reinversión en la gestión de la Oficina a la vez que funcionaba de reservorio a donde iban a parar muchos de los libros valiosos hasta ese momento en manos de propietarios privados.

La Oficina del Historiador aprobó otro mecanismo que garantizaba la captación del patrimonio bibliográfico que se ponía a la venta en la Plaza de Armas o El Navío y lo extendió a cualquier persona natural que poseyera un bien documental susceptible de ser considerado patrimonio, e incluso, antiguo, valioso o curioso. Se implementó entonces la compra a particulares por parte de la Biblioteca Histórica cuyo historial a la vanguardia de la gestión bibliográfica en La Ha- bana le había otorgado tal confiabilidad. Para ello se creó una comisión de valoración de los libros propuestos para su posible adquisición que desde entonces hasta la actualidad ha documentado el proceso de selección y adquisición y garantizando el crecimiento y actualización del fondo de todas las bibliotecas especializadas del Centro Histórico.

La posibilidad de adquirir libros para las bibliotecas se vinculó desde el principio a la Plaza de Armas y El Navío pues, tanto los bibliotecarios a cargo, como los especialistas de la librería y muchos libreros y anticuarios de la plaza, seleccionaban aquellas publicaciones que eran de interés de acuerdo con los años de publicación, utilidad temática, valores añadidos o intrínsecos o a la necesidad de completar colecciones. Del mismo modo, los especialistas de la Librería El Navío seleccionaban los libros antiguos que pudieran ser afines a estos criterios para que fueran adquiridos por la Oficina.

En muchos libreros de la Plaza de Armas, anticuarios y personal de El Navío se fue formando una sensibilidad -cuando no la tenían de antescapaz de reconocer los valores patrimoniales de los libros y la Oficina del Historiador proveyó el mecanismo para que pudieran engrosar el fondo bibliográfico de las bibliotecas.

La compra a través de los libreros, de El Navío o de personas naturales se complementó con la compra a instituciones cubanas y extranjeras que

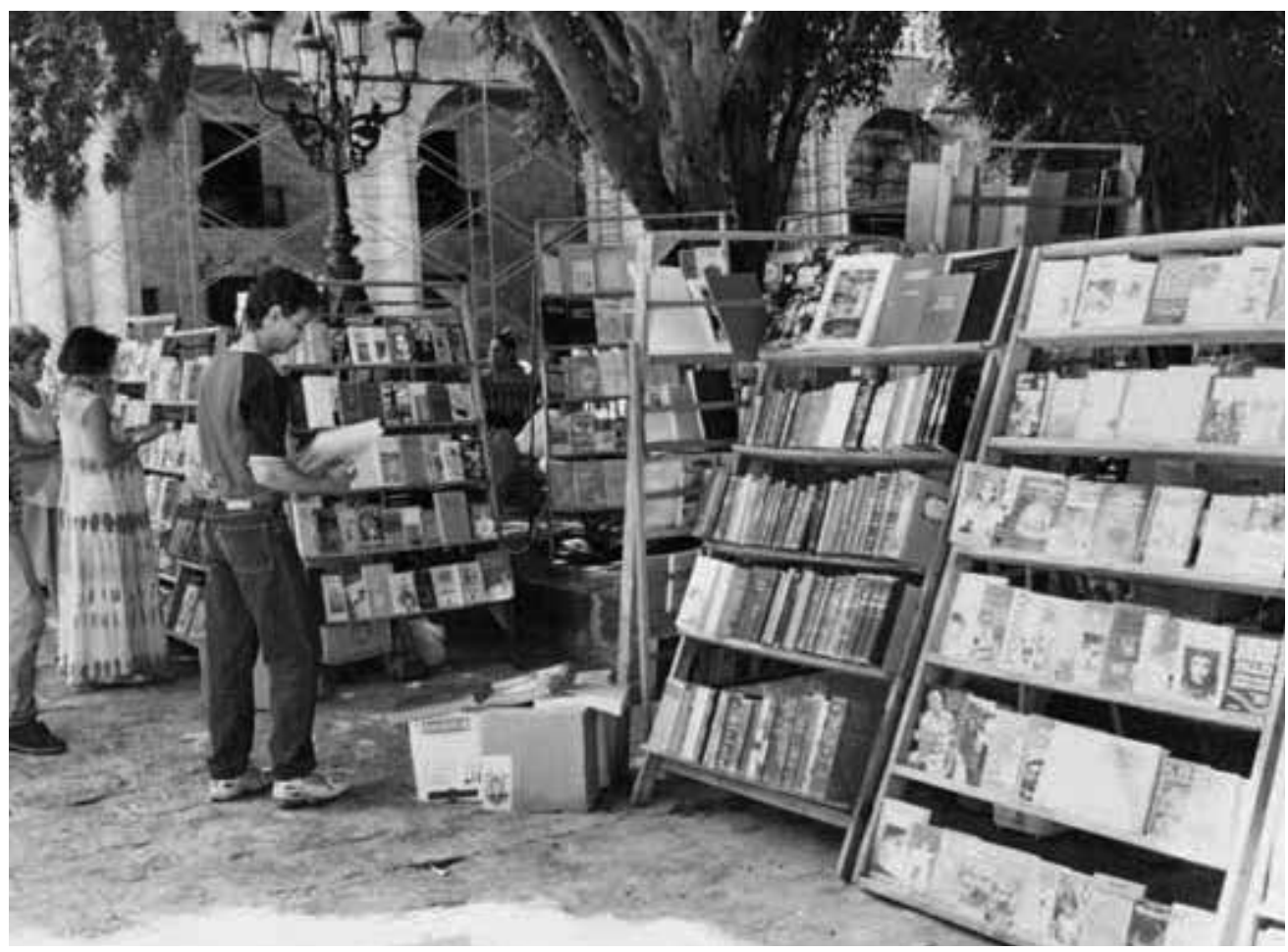

Figura 2:

Feria de Libros Viejos de la Plaza de Armas en la década de 1990. Fuente: Libreros 
garantizaran un fondo lo más actualizado posible dadas las circunstancias editoriales de Cuba y la complejidad de la importación de publicaciones extranjeras. Por esta vía se mantuvo actualizada la colección de revistas como National Geographic e Historia 16y se adquirieron títulos contemporáneos que complementaban los perfiles temáticos de las bibliotecas del Centro Histórico.

Gracias a esta gestión bibliográfica la cantidad de documentos comprados ha ido creciendo y de 187 que se compraron en 1999, en el año 2013 se llegaron a comprar $2221^{3}$.

Para lograr que este mecanismo funcionara, era necesario contar con el presupuesto necesario que pudiera ser empleado en adquirir libros de reciente publicación y libros raros, antiguos, valiosos o curiosos. Para este fin se destinó parte de los fondos recaudados gracias la nueva gestión iniciada en los noventa y del presupuesto estatal asignado a la Oficina del Historiador, y desde sus inicios se distinguieron las compras de libros contemporáneos en instituciones de las compras a personas naturales a través de las cuales llegaban los libros más raros o antiguos. Cada concepto se refleja en la distinción de partidas económicas cuyos fines están claramente diferenciados.

Hacia lo interno, en 1996 se creó la Organización Económica Museo de la Ciudad con personalidad jurídica propia y patrimonio inde- pendiente que fue la antesala de la que luego sería la Dirección General de Patrimonio Cultural, nombrada así desde 1998. Es justamente con esta Dirección que el patrimonio bibliográfico terminó de organizarse en la Oficina del Historiador y adquirió un grado de sistematicidad sin precedentes en su gestión y efectividad.

La Oficina potenció y extendió las bibliotecas en el Centro Histórico con la creación de más de una veintena de ellas para responder a las necesidades de información de instituciones especializadas, como museos y casas-museo. Retomando la filosofía iniciada por Emilio Roig en 1938 cuando fundó la Biblioteca Histórica Cubana y Americana, las bibliotecas se sumaron a la obra rehabilitadora desde el mundo de la información, pero también, como gestoras del patrimonio bibliográfico cuyo fondo se iba engrosando mediante donaciones y compras, y como depositarias de la producción bibliográfica contemporánea.

Las bibliotecas empezaron a proliferar en el Centro Histórico en los años ochenta y noventa pero con un carácter aislado y solo en función de servicios tradicionales ajustados únicamente al perfil temático de la entidad donde estaban enclavadas. Como resultado de tal expansión -incluso territorial-y de la necesidad de estructurar y sistematizar la gestión bibliográfica, habida cuenta de que son estas instituciones las que

1999 y del Informe anual de la Dirección de Patrimonio Documental en 2013.

3. Datos tomados de los informes publicados por la
Figura 3:

Biblioteca Ada Elba Pérez de la Casa de la Poesía.

Fuente:

Archivo personal Grisel Terrón

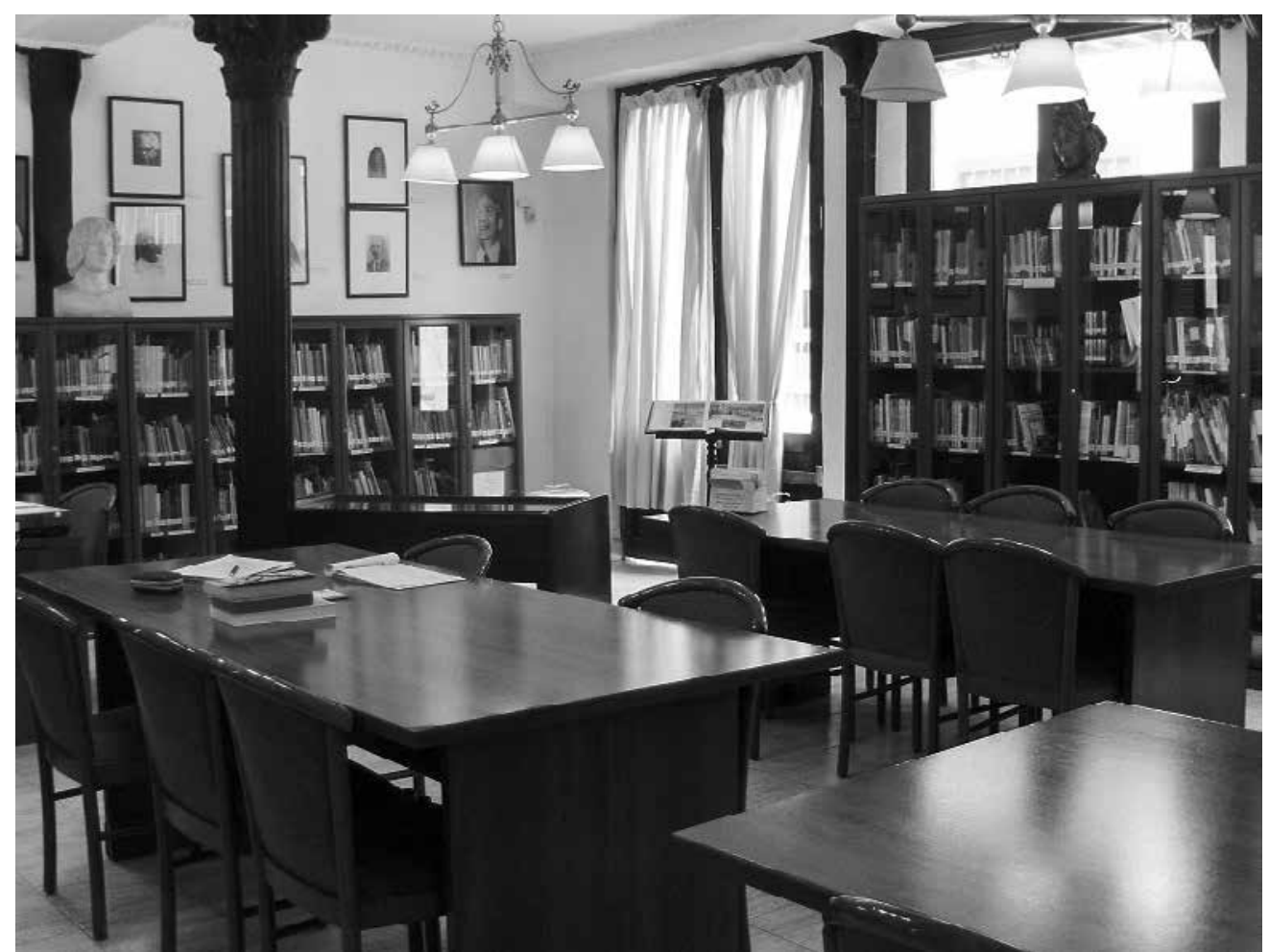




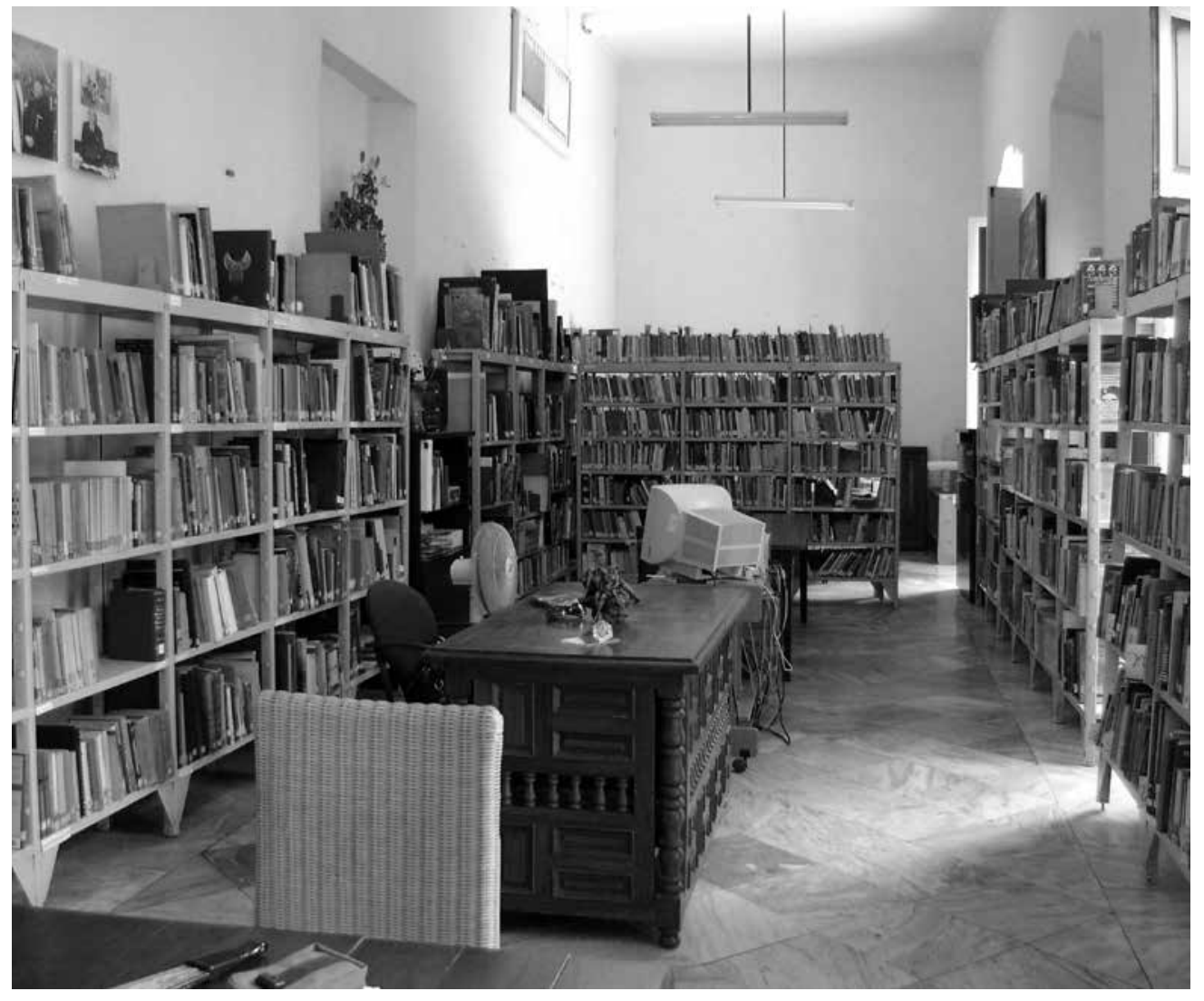

dentro de la Oficina del Historiador cumplen la función de preservar y gestionar de cara al futuro el patrimonio bibliográfico, en 2009 se creó la Subdirección de Bibliotecas de la Dirección de Patrimonio Cultural. La Subdirección nació para normalizar, organizar y coordinar toda la gestión bibliográfica y bibliotecaria, y para ello creó herramientas técnicas para la preservación y socialización del patrimonio bibliográfico de la Oficina. Todo esto, con la visión de lograr la preservación y la difusión bibliográfica usando políticas y estrategias de gestión novedosas y de gran alcance.

Por esta razón se incorporaron nuevas formas de difusión del patrimonio atesorado con las que se intentó trascender los límites tradicionales de los servicios bibliotecarios. En este sentido, antes de la creación de la Subdirección, la Biblioteca Histórica había incursionado en la gestión de difusión de su fondo al añadir a sus servicios la función expositiva y al iniciar la automatización y digitalización de su fondo con no pocas dificultades.

Los incisos d y e del artículo 6 del Decreto 143 de 1993 dieron al Historiador la posibilidad de buscar fuentes externas para contribuir al financiamiento de la rehabilitación y preservación del patrimonio cultural bajo su gestión. Así, se abrieron las puertas a la cooperación internacional para lo cual desde el inicio, “[...] se contó con la colaboración de la Agencia Española de Cooperación Internacional que transmitió sus experiencias profesionales, brindó ayuda económica y en recursos materiales para ese fin, también participaron varias entidades nacionales que realizaron valiosos aportes y contribuciones" (Volta, 2010, p. 8).

La Declaratoria de la Unesco en 1982 mediante la cual el Centro Histórico de La Habana entró a la lista del patrimonio mundial, constituyó una carta de presentación de la Oficina ante organismos internacionales, entidades no gubernamentales y hasta personas naturales sensibilizadas con el patrimonio y la cultura cubanos.

Para que la Oficina del Historiador gestionara la voluntad internacional en función del rescate patrimonial con su sello de desarrollo social, en febrero de 2002 se creó, como parte de la estructura de la Oficina, el Departamento de Cooperación Internacional, con la misión de coordinar, facilitar, gestionar y controlar las iniciativas por esta vía de financiamiento. Más tarde, la eficiente gestión de este Departamento lo convirtió en una Dirección Asesora del Historiador.
Figura 4:

Biblioteca Alfonso Reyes de la Casa Museo del Benemérito de las Américas Benito Juárez. Fuente: Archivo personal Grisel Terrón 


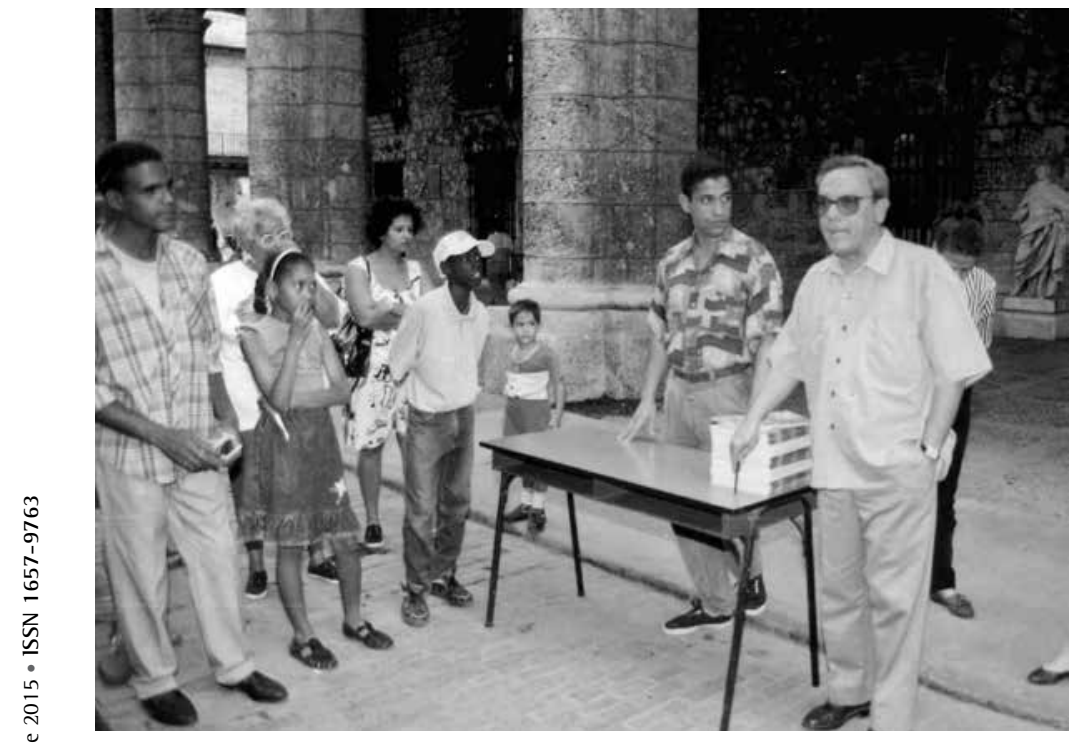

Figura 5:

Presentación del primer número de la revista Opus Habana, 1996.

Fuente:

Fototeca histórica

3. Catálogo disponible en http://catalogo. patrimonio.ohc.cu computadoras, impresoras y escáneres altamente especializados que ayudaron a desarrollar la estrategia para preservar, difundir y hacer accesible el patrimonio bibliográfico adquirido y conservado durante tantos años en las bibliotecas del Centro Histórico.

Gracias a la cooperación española y al proyecto interno de la Oficina del Historiador "Pangea" se logró automatizar el inventario y catalogación de todas las bibliotecas así como poner en línea el catálogo colectivo ${ }^{3}$, lo cual, sin dudas, marca un hito en la gestión del patrimonio bibliográfico de la institución. Por primera vez el acceso a los catálogos de las bibliotecas del Centro Histórico pudo hacerse desde cualquier lugar del mundo y se dieron los primeros pasos para dar acceso también a los fondos cuyos originales serían debidamente preservados y retirados de la manipulación, sin menoscabo de su consulta pública.

La Agencia Suiza de Cooperación para el Desarrollo (Cosude) también hizo su aporte a la socialización del patrimonio bibliográfico de la Oficina del Historiador al gestionar la donación de computadoras hecha por la ETH de Zúrich. Los equipos que llegaron a las bibliotecas por esta vía se destinaron mayoritariamente a la consulta de los usuarios.

En la gestión bibliográfica de una institución, no solo han de tenerse en cuenta sus esfuerzos y acciones por rescatar el patrimonio bibliográfico y por el mantenimiento de las bibliotecas a su cargo como entidades donde se deposita y difunde, sino también, su preocupación por la producción de un nuevo patrimonio, incluso a partir del ya atesorado, y por la creación de nuevas maneras de difundirlo: una especie de espiral que contiene y se genera de manera simultánea.

En la Oficina del Historiador el sistema de publicaciones nació junto a ella en la década de los años treinta y en el nuevo milenio se convirtió en poderosa vía de comunicación y difusión.

En pleno período especial, cuando las precariedades ensombrecían el panorama editorial en Cuba, aparecieron la revista Opus Habana y la Editorial Boloña bajo el sello de la Oficina del Historiador. Estas entidades institucionales surgieron para comunicar el patrimonio de una u otra forma, porque "comunicar el patrimonio es garantía de su preservación. Ni los decidores gubernamentales, ni los hacedores de la restau- 


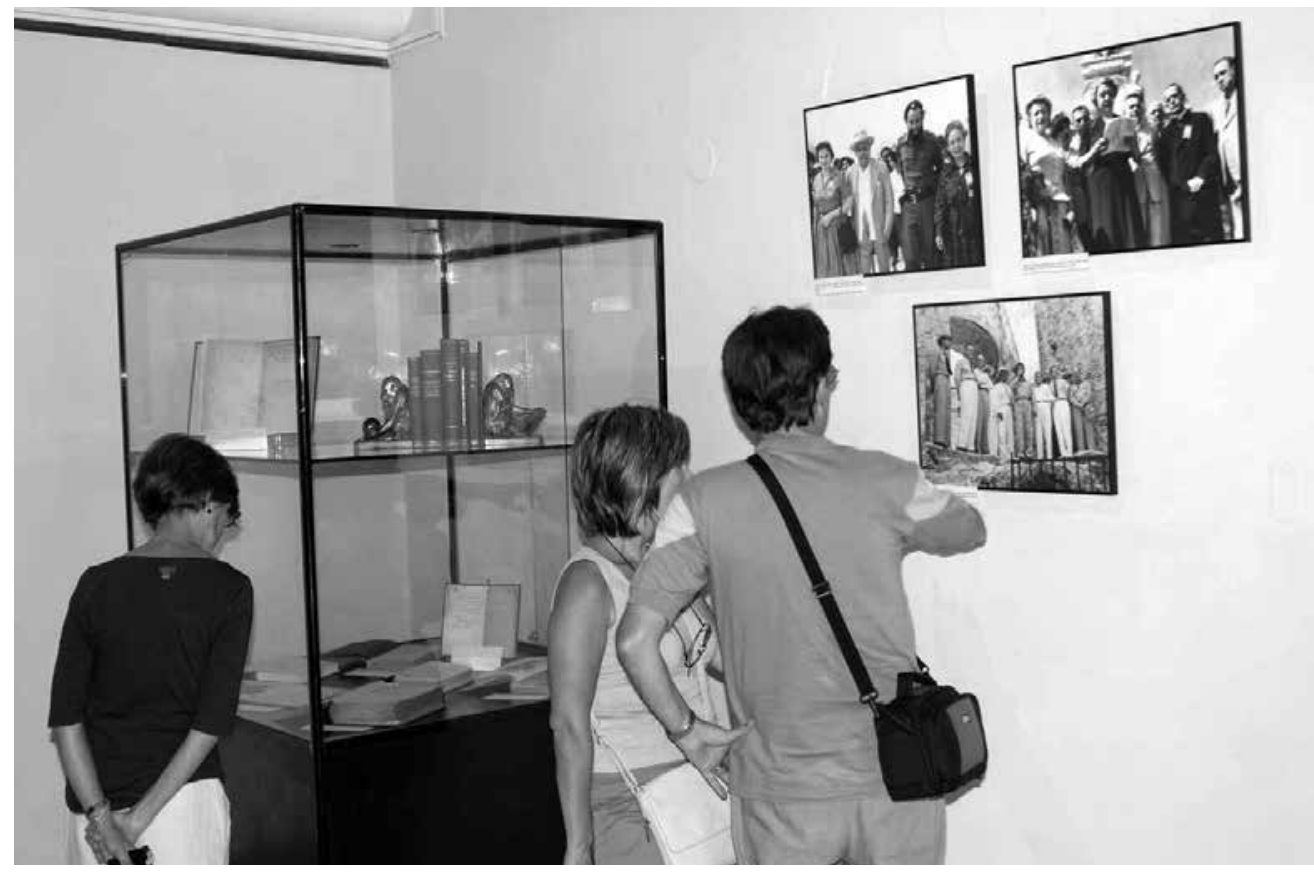

Figura 6:

Exposición bibliográfica "Para Emilio", 2006.

Fuente:

Archivo personal Grisel Terrón

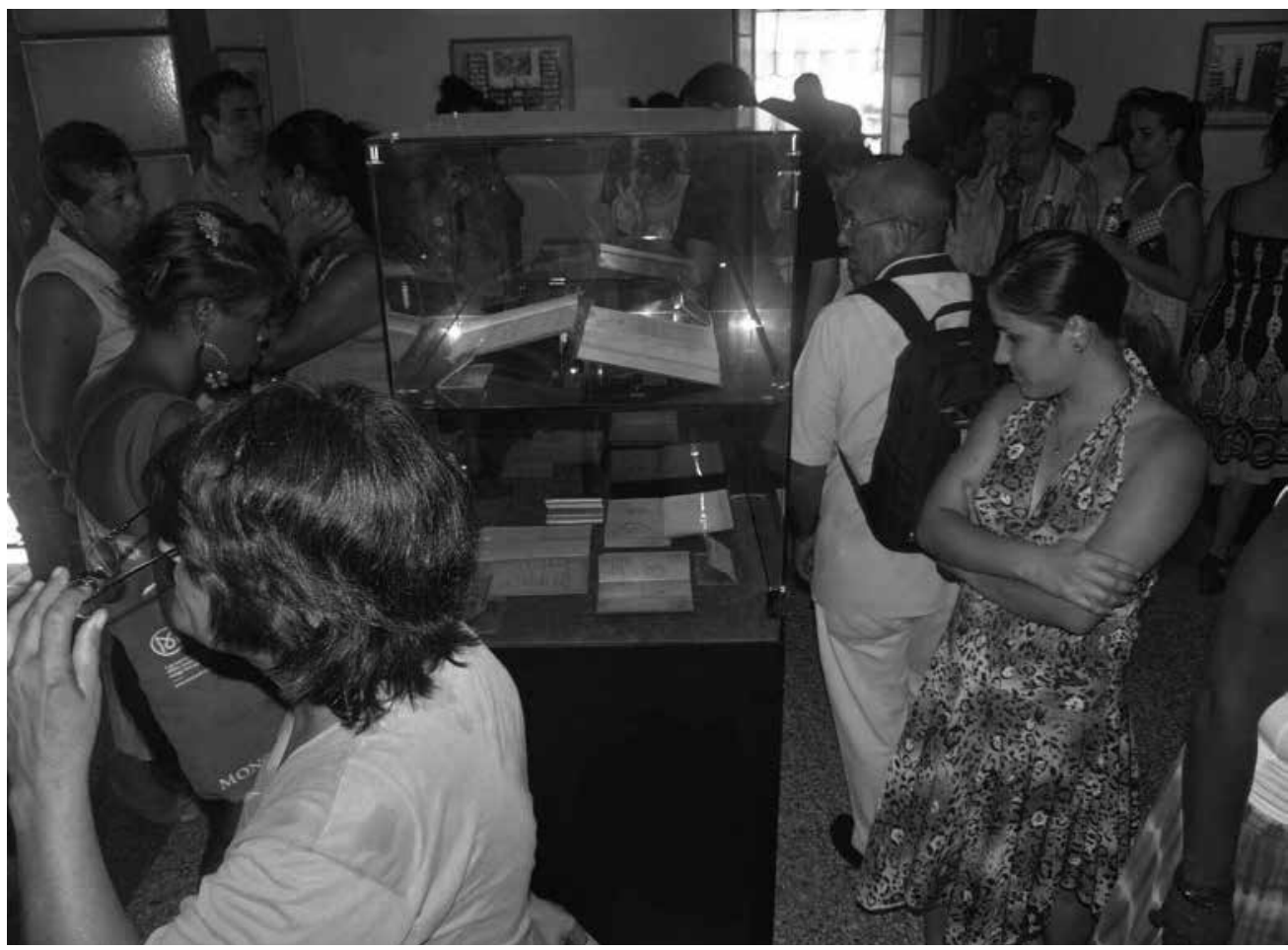

Figura 7:

Exposición por el aniversario de la Biblioteca Histórica, 2015.

Fuente:

Archivo personal Grisel Terrón

ración, ni los pobladores de un sitio -portadores ellos mismos del denominado Patrimonio intangible-, comprenderán su responsabilidad en un asunto que debiera interesarnos a todos, si no se apropian conscientemente de los valores a perpetuar. Y el sentido de pertenencia -es sabido- nace del reconocimiento" (García, 2013).

La revista Opus Habana apareció en 1996 con el objetivo de documentar la gesta restauradora del Centro Histórico, difundir la historia de la ciudad, divulgar la obra de destacadas personalidades cubanas y socializar el patrimonio conservado en los museos, bibliotecas y archivos institucionales. Una excelente factura, y el propio esquema de la revista, la distinguen y garantizan su sentido. Cada número es dedicado a entrevistar a una figura destacada de la cultura cubana; así se ha divulgado la obra de escritores, historiadores, artistas, restauradores, arquitectos, bibliotecarios, que han impactado de alguna ma- 


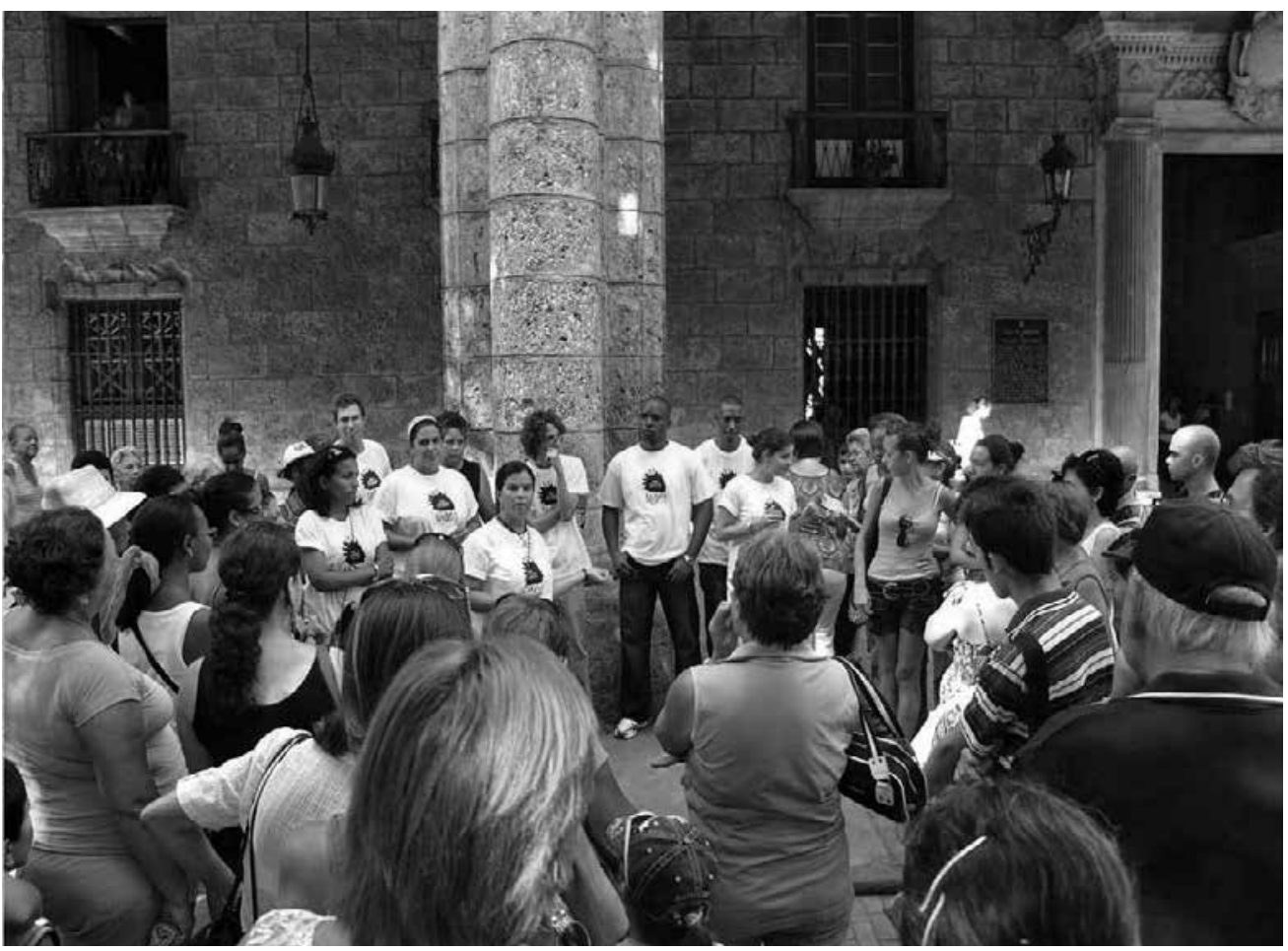

nera la cultura cubana o habanera. Igualmente, las portadas remiten a la obra de pintores contemporáneos quienes las realizan especialmente para cada número en el que luego se dedica un espacio al pintor en cuestión. La portada trasera contribuye a difundir el fondo documental de los archivos y bibliotecas de la Oficina pues en ella se publica una fotografía o grabado alegórico al tema del número. El breviario da cuenta de la gestión sociocultural del Centro Histórico mediante sucintas noticias de eventos relacionados con esta parte de la ciudad. Con esta filosofía, la revista Opus Habana rescata el pasado y presente para el futuro.

Asimismo, la Editorial Boloña -que debe su nombre a la reconocida familia impresores de La Habana colonial-, surgió un año después que Opus Habana, en 1997, con la misión de divulgar y preservar el patrimonio histórico, fotográfico, documental e intangible de la ciudad y, en especial, de su Centro Histórico. Este sello editorial cuenta actualmente con más de un centenar de publicaciones dedicadas a temas históricos y relacionados con el patrimonio cultural.

De la misma manera, la integración de de papel” (2004), "La prensa en el tiempo" (2005),

"Para Emilio" (2006)

"Del trazo a la sonrisa" (2010) y “Homenaje a Emilio Roig” (2014). sobre todo desde finales de los noventa y con más fuerza, a partir del año 2000 cuando se va conformando una visión renovada de lo que es la gestión bibliográfica que no menosprecia fórmulas para que el patrimonio bibliográfico gane su condición a partir de la identificación de los públicos con él.

La Oficina del Historiador, consciente de que socializar el patrimonio es un modo de preservarlo, ha promovido exposiciones bibliográficas cuyo discurso expositivo pretende poner en evidencia la relevancia de textos que podrían pasar desapercibidos en un catálogo. En la primera década de los años 2000 se retomó este estilo de difusión que formó parte de la gestión bibliográfica primigenia en la Oficina del Historiador.

En 2002 la exposición "Lugar donde se cura el alma” se realizó de manera conjunta con el Archivo y la Fototeca históricos en el marco de la Primera Jornada Bibliotecaria en el Centro Histórico. La intención de la exposición, que tuvo su sede en el Museo de la Ciudad, era mostrar parte de los tesoros documentales conservados o recuperados durante años a través de los mecanismos antes descritos. A esta exposición han seguido otras ${ }^{4}$ que, organizadas por temáticas o tipo documental, han contribuido a socializar publicaciones conservadas en las bibliotecas y a destacar sus valores y detalles. 


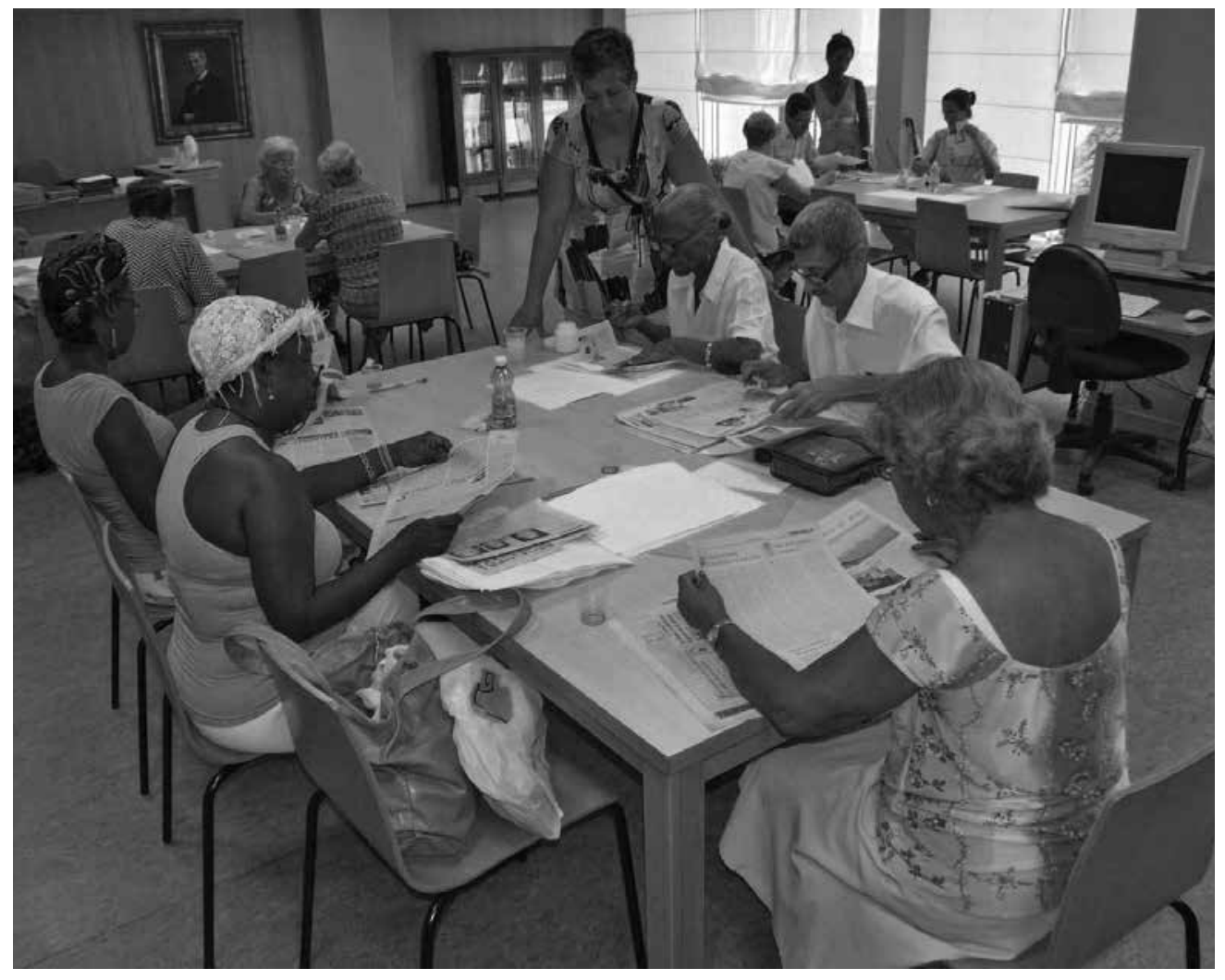

En el afán por explotar todas las vías posibles para comunicar y socializar el patrimonio bibliográfico, las bibliotecas generan actividades de extensión que trascienden los servicios tradicionales. Entre ellas se destacan los talleres de verano, las actividades por la semana del libro infantil cubano y, sobre todo, la inserción en el programa cultural de verano Rutas y Andares, la Jornada Bibliotecaria y la inclusión de salas expositivas permanentes en centros culturales de última generación.

Rutas y Andares funciona en la Oficina del Historiador como un programa de puertas abiertas al público en general, donde se muestran colecciones y objetos reubicados didácticamente, y también el trabajo que se realiza en el centro histórico con el patrimonio. La participación de las bibliotecas en este programa se inició con un "Andar por los exlibris" en los primeros años del 2000. Durante años el Andar con el Bibliotecario -título genérico con el que se nombra este Andar-, se mantuvo entre los preferidos por el público, razón por la que se ha mantenido en casi todas las ediciones. Las temáticas programadas han abarcado desde las publicaciones valiosas, raras o curiosas y los elementos que valorizan a los libros - como el caso mencionado de los exlibris- hasta temáticas más complejas como las bibliotecas digitales en el año 2011.

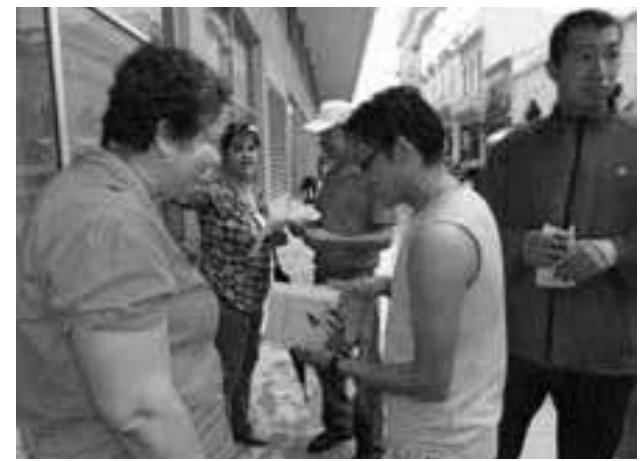

Figura 9:

Rutas y Andares. Taller de verano "Las colecciones facticias", 2015.

Fuente:

Archivo personal Grisel Terrón

Los 7 de junio se celebra el Día del Bibliotecario Cubano en memoria de Antonio Bachiller y Morales, y tal celebración se ha extendido a una semana de actividades en el Centro Histórico cuyo fin es promover y socializar el patrimonio bibliográfico y la labor de los bibliotecarios. Cada año se organiza un programa de actividades dirigidas a públicos diversos, pero la más democrática de todas ellas es la campaña que se ha incluido en los dos últimos años: en 2013 bajo el nombre "El Bibliotecario te regala un libro" y en 2014 y 2015 con el título "Libros en movimiento". Esta campaña pretende una socialización absolutamente participativa donde no exista condición alguna para tener un libro: ese día los bibliotecarios regalan libros a los que visiten el Centro Histórico.
Figura 10:

Campaña "El

Bibliotecario te regala un libro", 2013.

Fuente:

Archivo personal

Grisel Terrón 


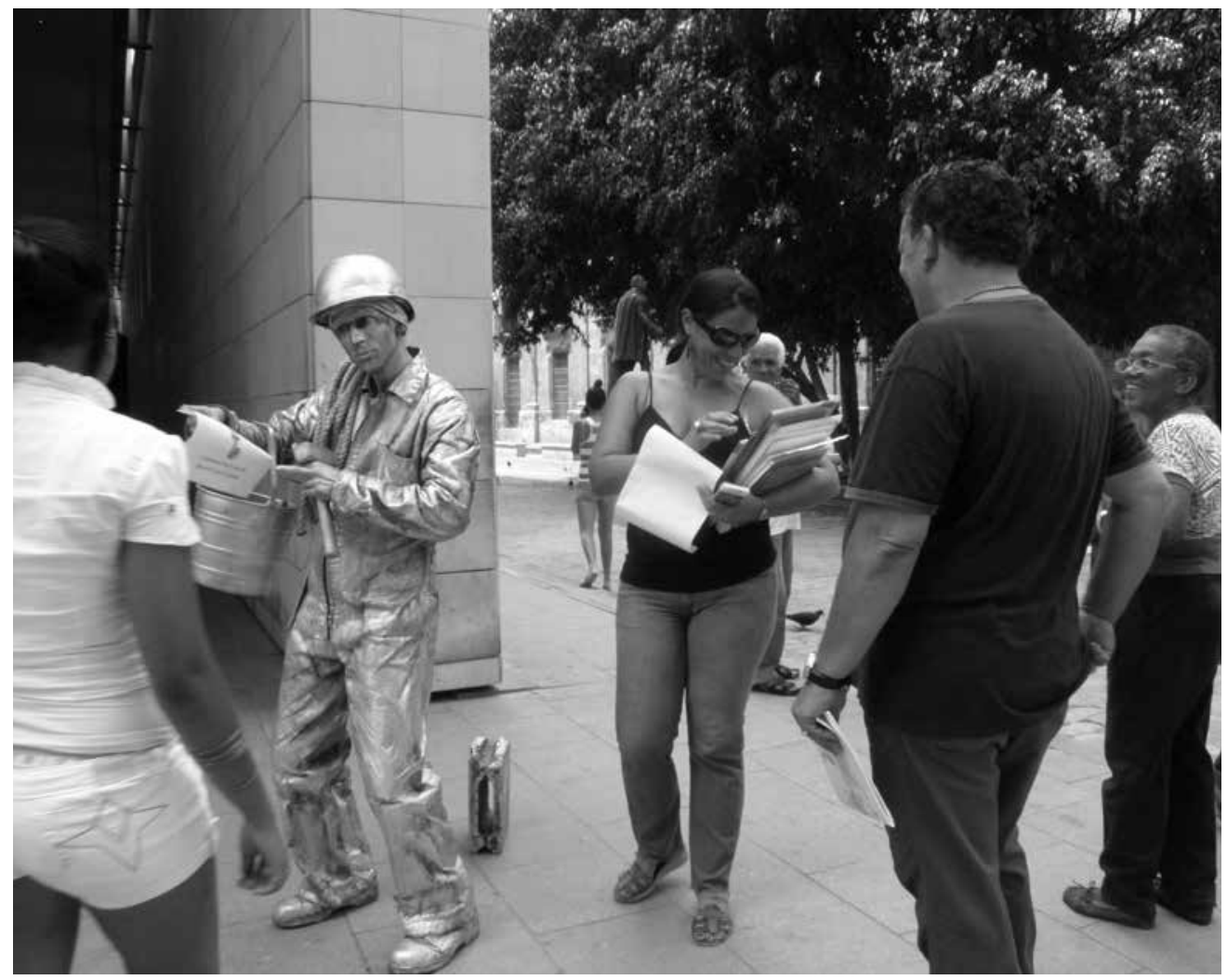

Figura 11: Campaña "Libros en Movimiento", 2014.

Fuente:

Archivo personal Grisel Terrón

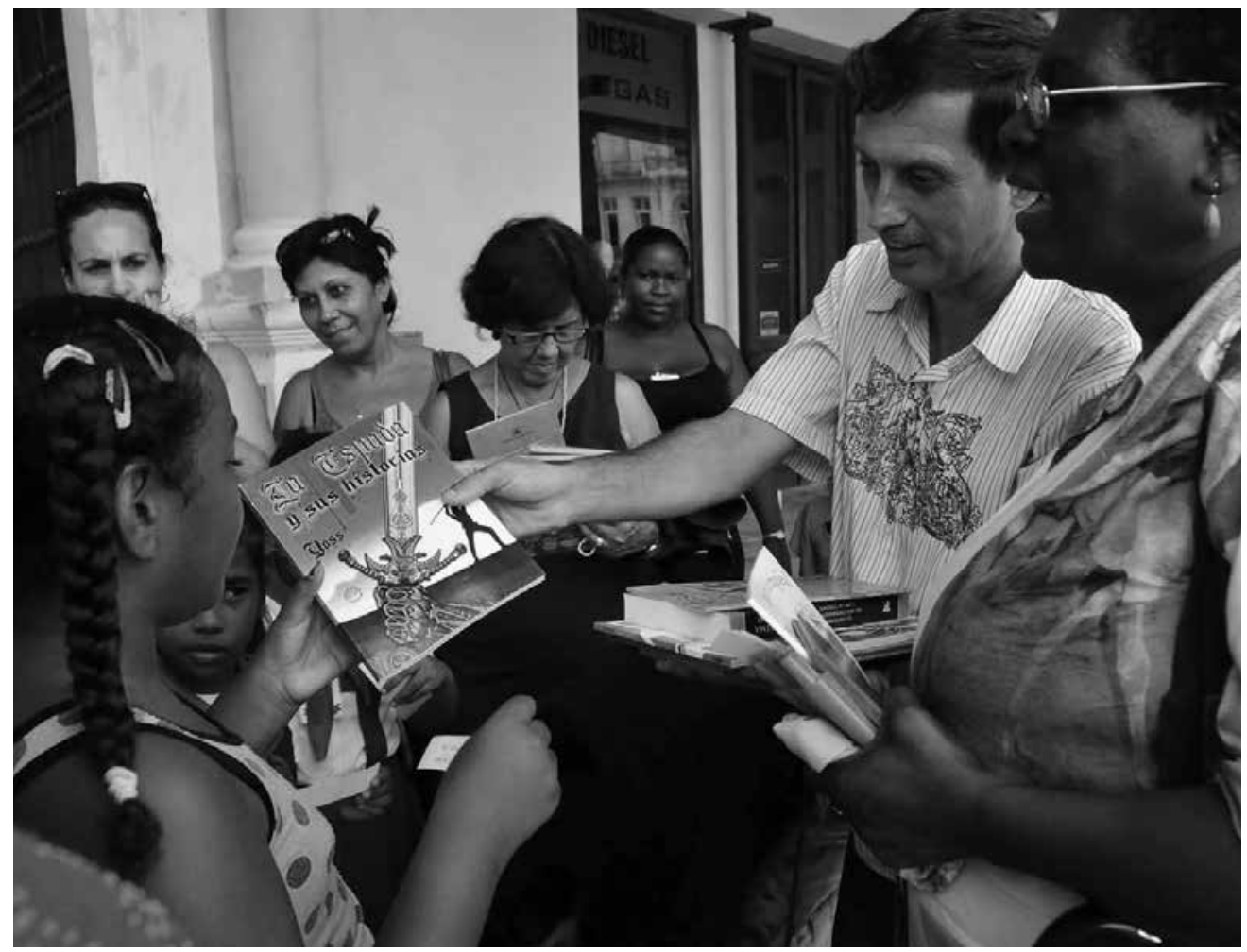

Desde 2012 se proyecta una sala dedicada al libro cubano en el otrora Palacio del Segundo Cabo donde ahora se diseña un Centro de interpretación de las relaciones entre Cuba y Europa. Con la cooperación de la Unión Europea y la
Unesco, por primera vez se dedicará al libro una sala expositiva permanente donde, a través de técnicas interpretativas y modernas tecnologías, se mostrará la historia del libro en Cuba en la relación histórica de este país con Europa. 


\section{Conclusiones}

El proverbio chino que asocia crisis con oportunidad, ha encontrado su validación en la gestión bibliográfica de la Oficina del Historiador. La convulsa crisis que marcó a los años noventa con el período especial, soltó las amarras de un proyecto de luz propia y plantó bandera en el panorama cultural cubano donde el rescate y socialización del patrimonio bibliográfico han sido una línea directriz. El enfoque transdisciplinar de la gestión bibliográfica que aprovecha las potencialidades de un modelo de gestión sui genéris ha contribuido, de manera quizás invaluable, a la cultura literaria cubana a partir de la obra regeneradora de la Oficina del Historiador de la Habana.

\section{Referencias}

Cuba, Ministerio de Justicia (1993). Decreto Ley 143/1993. Gaceta Oficial de la República de Cuba No. 14 Ordinaria de 4 de noviembre de 1993.
García, A. L. (2013). Comunicar el Patrimonio: un desafío del siglo XXI. La Jiribilla, año XIl. Recuperado de http://www.lajiribilla. cu/articulo/5056/comunicar-el-patrimonioun-des...

Leal, E. (2009). Legado y memoria. La Habana: Ediciones Boloña.

Oficina del Historiador de la Ciudad de La Habana (1999). Informe de trabajo del año 1999.

Oficina del Historiador de la Ciudad de La Habana (2009). Proyecto Acceso al patrimonio documental de la nación que se atesora en el Centro Histórico de La Habana [2009-2011].

Oficina del Historiador de la Ciudad de La Habana (2013). Informe anual del año 2013. Dirección de Patrimonio Documental.

Oficina del Historiador de la Ciudad de La Habana. Plan Maestro (1996). Viaje en la memoria: apuntes para un acercamiento a La Habana Vieja.

Volta, Francisco (2010). Ciudades cubanas del patrimonio cultural. Contexto jurídico nacional actual. Un acercamiento al tema [Documento en pdf]. 